\title{
MK2 puts an additional brake on RIPK1 cytotoxic potential
}

\author{
Yves Dondelinger ${ }^{1,2} \cdot$ Tom Delanghe ${ }^{1,2} \cdot$ Mathieu M.J. Bertrand $\mathbb{( I}^{1,2}$
}

Received: 4 October 2017 / Accepted: 11 October 2017 / Published online: 9 January 2018

(c) ADMC Associazione Differenziamento e Morte Cellulare 2018

The kinase RIPK1 has received a lot of attention in the past recent years due to its ability to transduce a death signal whose consequences have been implicated in the pathogenesis of various diseases in the mouse [1]. RIPK1 was first believed to specifically trigger a regulated form of necrosis termed necroptosis, but later studies demonstrated that its enzymatic activity can promote apoptosis as well as necroptosis depending on the cellular context [2]. The fact that RIPK1 kinase inactive mice are viable and reach adulthood without developing any spontaneous overt phenotype indicates that RIPK1 kinase-dependent death is not a critical process for development, or tissue homeostasis in non-challenged conditions. This contrasts with the predominant prosurvival platform function of RIPK1 that is demonstrated by the perinatal lethality of the RIPK1deficient mice [3]. Thus, while the scaffold role of RIPK1 is needed for development/survival, the physiological necessity of RIPK1 kinase-dependent death has remained enigmatic. It is also unclear what regulates the switch between RIPK1 survival/ death functions and, consequently, the cause(s) of the pathologic RIPK1 activation in diseases. Three independent groups, including ours, just provided partial answers to these questions by demonstrating that RIPK1 kinase activation and cell death is negatively regulated by MK2-mediated phosphorylation, and that this protective mechanism is inhibited in a cellular model of Yersinia infection and in cells exposed to tumor necrosis factor (TNF) and TWEAK [4-6].

Although identified downstream of various innate immune receptors, RIPK1 is most extensively studied in the

Edited by G. Melino

Mathieu M.J. Bertrand

mathieu.bertrand@irc.vib-ugent.be

1 VIB Center for Inflammation Research, Technologiepark 927, 9052 Zwijnaarde-Ghent, Belgium

2 Department of Biomedical Molecular Biology, Ghent University, Technologiepark 9279052 Zwijnaarde-Ghent Belgium
TNFR1 pathway. Upon TNF binding, RIPK1 is recruited to TNFR1 complex I where its ubiquitylation by cIAP $1 / 2$ and LUBAC contributes to the induction of a prosurvival/ proinflammatory response by activation of the mitogenactivated protein kinase (MAPKs) and NF-KB pathways [7]. RIPK1 kinase-dependent cell death is another possible outcome of TNFR1 activation. It requires dissociation of RIPK1 from complex I and its subsequent enzymaticdependent association with FADD to form the cytosolic death-inducing complex IIb. Death is, however, not the default response of most cells to TNF, indicating existence of mechanisms actively suppressing it [7]. We previously revealed that phosphorylation of RIPK1 by IKK $\alpha / \beta$ prevents RIPK1 kinase-dependent cell death [8]. In line with these findings, in vivo conditions affecting proper IKK $\alpha / \beta$ activation were shown to result in pathologies caused by excessive RIPK1 kinase-dependent cell death [8-10].

Three independent groups now demonstrate that MK2, a substrate of p38 MAPK, additionally represses the prodeath function of RIPK1 [4-6]. The studies agree on the fact that MK2 interacts with and directly phosphorylates RIPK1 to limit its activation and the subsequent assembly of complex IIb. They show that RIPK1 is phosphorylated by MK2 on Ser321 and Ser336 (Ser320 and Ser335 in human), but the respective role of these residues in the direct or indirect regulation of RIPK1 kinase activity will require further investigation. Repression of RIPK1 kinase-dependent cell death by phosphorylation on Ser321 and Ser336 has also just been described in two additional studies, but the authors curiously report phosphorylation of RIPK1 by the upstream kinase TAK1 or by $\operatorname{IKK} \beta[11,12]$. In the latter case, the discrepancy with our results may originate from the fact that their conclusion was drawn using of the IKK inhibitor TPCA-1, which appears to have some inhibitory activity toward MK2. Importantly, phosphorylation of RIPK1 by $\mathrm{IKK} \alpha / \beta$ and $\mathrm{MK} 2$ are functionally distinct processes since MK2 inhibition further sensitize cells to TNF-mediated cell death in IKK $\alpha / \beta$ inactivated conditions. MK2 does, however, not regulate the most critical brake in the pathway but rather controls an additional layer of regulation limiting the extent of cell death. Indeed, inactivation of MK2 is not 

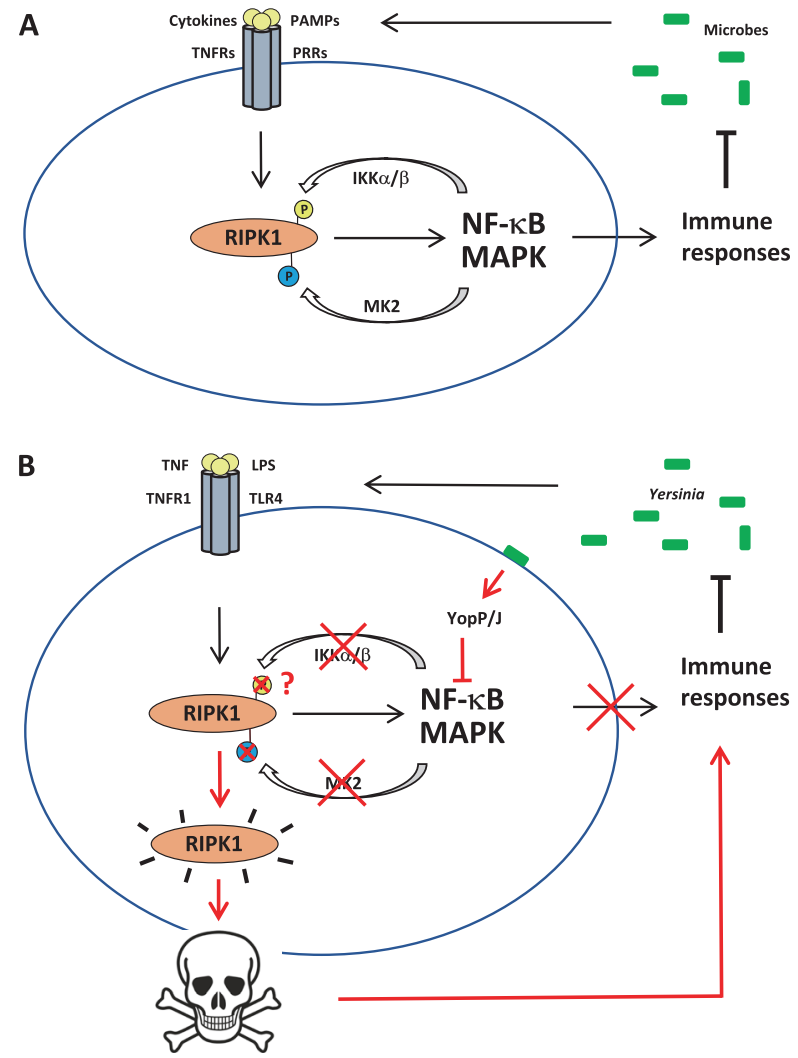

4 Fig. 1 Models of RIPK1 regulation by IKK $\alpha / \beta$ and MK2 phosphorylation. a RIPK1 regulates signaling downstream of several innate immune receptors. Cell death is generally not the default response of these receptors. Upon ligand binding, RIPK1 is recruited to the receptor signaling complex where its platform function contributes to the activation of the NF- $\mathrm{KB}$ and/or MAPK pathways for prosurvival and proinflammatory gene expression. The feedback phosphorylation of RIPK1 by IKK $\alpha / \beta$ and MK2 maintains RIPK1 in a prosurvival mode. b Certain pathogens, such as the Yersinia genus of bacteria, express inhibitors (YopP/J) of the NF- $\mathrm{KB}$ and MAPK pathways in an attempt to evade host defenses. Under these conditions, RIPK1 is not properly phosphorylated by $\mathrm{IKK} \alpha / \beta$ and $\mathrm{MK} 2$, which leads to its kinase activation and results in apoptosis. The cell demise generates signals that activate anti-bacterial defenses. c. Certain members of the TNF superfamily, such as TWEAK, induce degradation of a pool of cIAP1/2 to activate the non-canonical NF- $\kappa$ B pathway. The reduced levels of cIAP1/2 in the cell affects proper activation of the MAPK and canonical NF- $\kappa \mathrm{B}$ pathways by TNF. As a result, RIPK1 phosphorylation by $I K K \alpha / \beta$ and MK2 is affected and the cell succumb by RIPK1 kinase-dependent cell death

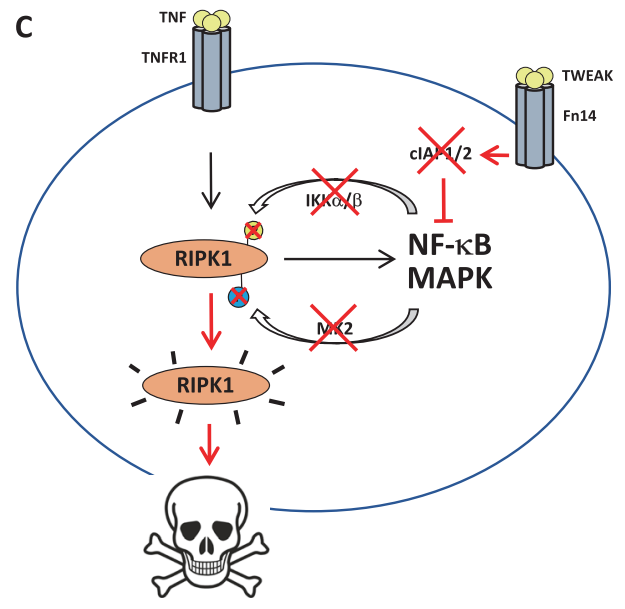

sufficient to switch the TNFR1 response from survival to death. Regulation of RIPK1 by IKK $\alpha / \beta$ and MK2 are also spatially distinct phenomena. MK2 phosphorylates a cytoplasmic pool of RIPK1 that presumably subsequently integrates complex I, while IKK $\alpha / \beta$ phosphorylate RIPK1 directly in complex I $[4,6]$. It will be interesting to evaluate in the future if cytosolic phosphorylation of RIPK1 by MK2 can prevent its direct integration into complex IIb.

In addition to TNFR1, RIPK1 regulates signaling downstream of other immune receptors, including CD95, DR5, TLR-3/4, and RIG-I [3]. Even though these receptors have the potential to trigger cell death, cell demise is

generally not their default response. The immune receptors employing RIPK1 converge in the activation of the NF- $\mathrm{KB}$ and MAPKs pathways for the induction of inflammatory mediators. It is currently unknown whether RIPK1 is phosphorylated by IKK $\alpha / \beta$ and MK2 downstream of all these receptors, but RIPK1 is at least also phosphorylated by MK2 upon LPS stimulation [4, 5]. It is, therefore, tempting to speculate that RIPK1 kinase-dependent death could have evolved as a backup mechanism aimed at eliminating cells unable to mount a proper immune response. When the IKK $\alpha / \beta$ and $\mathrm{p} 38 / \mathrm{MK} 2$ pathways are properly activated, each signaling branch tags RIPK1 by phosphorylation to keep it in a prosurvival mode (Fig. 1a). In contrast, when these pathways are not properly activated, the absence of tags results in RIPK1 activation and in the elimination of the cell. Many pathogen deliver virulence factors into host cells to evade host defenses. This is, for example, the case of the Yersinia genus of bacteria that inject an acyltransferase, named YopJ/P, capable of inhibiting the catalytic activity of TAK1 and thereby affecting the activation of both the IKK $\alpha / \beta$ and MK2 signaling branches. Remarkably, Menon et al. [4] demonstrated that infection of macrophages with Yersinia enterocolitica affects MK2 phosphorylation of RIPK1 and results in RIPK1 kinase-dependent apoptosis (Fig. 1b). Importantly, the in vivo relevance of these findings is provided by another group who just demonstrated that Yersinia-induced RIPK1 kinase-dependent apoptosis is critical for host survival [13]. RIPK1 kinase-dependent death is proposed to contribute to the innate immune response by providing a cell extrinsic signal required for proinflammatory cytokines production and anti-bacterial defense [13].

Apart from the infectious context, genetic and/or environmental perturbations may also affect proper activation of 
the IKK $\alpha / \beta$ and p38/MK2 MAPK pathways, and consequently result in RIPK1 hypo-phosphorylation, RIPK1 kinase-dependent death, and, in certain cases, development of inflammatory diseases caused by excessive cell death induction. We found that co-sensing of TNF with TWEAK resulted in RIPK1 kinase-dependent death by partially affecting the IKK $\alpha / \beta$ and MK2 checkpoints (Fig. 1c), which could be amplified by further inhibition of these kinases pharmacologically [6]. Indeed, certain members of the TNF superfamily, including TWEAK and CD40L, activate the non-canonical NF- $\mathrm{B}$ pathway by inducing degradation of a pool of cIAP1/2. In light of the role of cIAP1/2-mediated RIPK1 ubiquitylation for activation of both the NF- $\mathrm{B}$ and MAPKs pathways, we found that TWEAK co-stimulation impaired TNF-dependent activation of IKK $\alpha / \beta$ and MK2. Consequently, TWEAK partially affected phosphorylation of RIPK1 by IKK $\alpha / \beta$ and MK2, and resulted in RIPK1 kinase-dependent cell death. It is interesting to speculate that a similar scenario could take place in inflammatory situations where cells are presumably exposed to multiple TNF superfamily members, such as in the in vivo TNFinduced shock model. It is also interesting to notice that TWEAK and CD40L are upregulated in inflammatory bowel disease and rheumatoid arthritis [14, 15], two TNFdriven human pathologies for which RIPK1 kinase inhibitors may turn very promising.

Altogether, these recent findings shed light on the importance of RIPK1 phosphorylation by IKK $\alpha / \beta$ and MK2 to keep RIPK1 prodeath function in check, and provide insights on the physiological role of RIPK1 kinasedependent cell death during bacterial infection and inflammation.

\section{Compliance with ethical standards}

Conflict of interest The authors declare that they have no competing interests.

\section{References}

1. Linkermann A, Green DR. Necroptosis. N. Eng. J. Med. 2014;370:455-65.

2. Dondelinger Y, et al. RIPK3 contributes to TNFR1-mediated RIPK1 kinase-dependent apoptosis in conditions of cIAP $1 / 2$ depletion or TAK1 kinase inhibition. Cell Death Differ. 2013;20:1381-92.

3. Silke J, et al. The diverse role of RIP kinases in necroptosis and inflammation. Nat Immunol. 2015;16:689-97.

4. Menon MB, et al. p38MAPK/MK2-dependent phosphorylation controls cytotoxic RIPK1 signalling in inflammation and infection. Nat Cell Biol. 2017;19:1248-59.

5. Jaco I, et al. MK2 phosphorylates RIPK1 to prevent TNF-induced cell death. Mol Cell. 2017;66:698-710. e695.

6. Dondelinger Y, et al. MK2 phosphorylation of RIPK1 regulates TNF-mediated cell death. Nat Cell Biol. 2017;19:1237-47.

7. Ting AT, Bertrand MJ. More to life than NF-кB in TNFR1 signaling. Trends Immunol. 2016;37:535-45.

8. Dondelinger $\mathrm{Y}$, et al. NF- $\mathrm{KB}$-independent role of $\mathrm{IKK} \alpha / \mathrm{IKK} \beta$ in preventing RIPK1 kinase-dependent apoptotic and necroptotic cell death during TNF signaling. Mol Cell. 2015;60:63-76.

9. Vlantis K, et al. NEMO prevents RIP Kinase 1-mediated epithelial cell death and chronic intestinal inflammation by NF-кB-dependent and -independent functions. Immunity. 2016;44:553-67.

10. Koppe $\mathrm{C}$, et al. IкB kinase $\alpha / \beta$ control biliary homeostasis and hepatocarcinogenesis in mice by phosphorylating the cell-death mediator receptor-interacting protein kinase 1. Hepatology. 2016;64:1217-31.

11. Mohideen F, et al. Quantitative phospho-proteomic analysis of $\mathrm{TNF} \alpha / \mathrm{NF} \kappa \mathrm{B}$ signaling reveals a role for RIPK1 phosphorylation in suppressing necrotic cell death. Mol Cell Proteomics. 2017;16:1200-16.

12. Geng J, et al. Regulation of RIPK1 activation by TAK1-mediated phosphorylation dictates apoptosis and necroptosis. Nat Commun. 2017:8:359.

13. Peterson LW, et al. RIPK1-dependent apoptosis bypasses pathogen blockade of innate signaling to promote immune defense. J Exp Med. 2017;214:3171-3182.

14. Croft M, Siegel RM. Beyond TNF: TNF superfamily cytokines as targets for the treatment of rheumatic diseases. Nat Rev Rheumatol. 2017;13:217-33.

15. Kawashima $R$, et al. Interleukin-13 damages intestinal mucosa via TWEAK and Fn14 in mice-a pathway associated with ulcerative colitis. Gastroenterology. 2011;141:2119-29. e2118. 